\title{
Investigating urinary tract infections in children
}

This Rational Imaging article by A Davis and colleagues contained some errors (BMJ 2013;346:e8654, doi:10.1136/bmj. e8654). In figure 1 the right hand label is wrong: the two arrows point to the "dilated lower pole [not "upper pole" as published] collecting system"; the legend below the figure is correct.

Additionally, in the online version (but not the print version) we made two further mistakes: we gave the wrong email address for the corresponding author (the correct address is adavis@doctors.org.uk), and we omitted the following statement: "AD is a part time, associate editor of the BMJ. She had no role in the assessment of this article for publication."

Cite this as: BMJ 2013;346:f1510

๑ BMJ Publishing Group Ltd 2013 